\title{
A pilot audit of a protocol for ambulatory investigation of predicted low-risk patients with possible pulmonary embolism
}

\author{
${ }^{1} \mathrm{AH}$ McDonald, ${ }^{2} \mathrm{R}$ Murphy \\ ${ }^{1}$ ACCS (Acute Medicine) Trainee in Department of Emergency Medicine, Royal Infirmary of Edinburgh; ${ }^{2}$ Consultant in Department of \\ Emergency Medicine and Acute Medicine, Royal Infirmary of Edinburgh, UK
}

\section{ABSTRACT}

Background: Patients with possible pulmonary embolism (PE) commonly present to acute medical services. Research has led to the identification of lowrisk patients suitable for ambulatory management. We report on a protocol designed to select low-risk patients for ambulatory investigation if confirmatory imaging is not available that day.

Methods: The protocol was piloted in the Emergency Department and Medical Assessment Area at the Royal Infirmary of Edinburgh. We retrospectively analysed electronic patient records in an open observational audit of all patients managed in the ambulatory arm over five months of use.

Results: We analysed 45 patients' records. Of these, 91.1\% required imaging to confirm or refute $\mathrm{PE}, 62.2 \%$ received a computed tomography pulmonary angiogram (CTPA). In $25 \%$ of patients, PE was confirmed with musculoskeletal pain (22.7\%), and respiratory tract infection (I5.9\%) the next most prevalent diagnoses. Alternative diagnoses was provided by CTPA in $32 \%$ of cases. We identified no adverse events or readmissions but individualised follow-up was not attempted. Conclusions: The data from this audit suggests this protocol can be applied to select and manage low-risk patients suitable for ambulatory investigation of possible PE. A larger prospective comparative study would be required to accurately define the safety and effectiveness of this protocol.

KEYWORDS Ambulatory, pulmonary embolism, protocol, investigation, outpatient
Correspondence to $\mathrm{AHJ}$ McDonald, Department of Medicine, Borders General Hospital, Melrose TD6 9BS, UK

tel. $+44(0) 7815750149$

e-mail

alexander.mcdonald@nhs.net

DECLARATION OF INTERESTS No conflict of interests declared..

\section{INTRODUCTION}

Pulmonary embolism (PE) is a common condition that presents to acute medical services with an estimated incidence of 95 episodes per 100,000 patient-years within the European Union.' Referral and presentation to secondary care accounted for approximately $5 \%$ of referrals to Acute Medicine in Southampton in 2006. ${ }^{2}$ Potentially a fatal condition, PE also carries an estimated in-patient mortality of I5.3\% at three months. ${ }^{3}$ Traditional management of possible PE has involved hospital admission and treatment with unfractionated heparin, pending imaging to confirm or refute the diagnosis.

In recent years, there has been increasing interest in ambulatory care, promoted by patient satisfaction ${ }^{4}$ and reduced costs of admission, both in economic terms (the cost of a Scottish acute medical bed in 2006-7 is quoted at $£ 483$ per day ${ }^{5}$ ) and complications of admission such as hospital acquired infection. The possibility of ambulatory management of PE has arisen since the advent of low molecular weight heparins (LMWH) (with administration and monitoring benefits over unfractionated heparin), building on ambulatory management of deep vein thrombosis. Publications by Kovacs in 2006 and Wells in 200 $\mathrm{I}^{7}$ suggested that approximately $50 \%$ of patients treated for PE could be managed as outpatients. In 2003, the British Thoracic Society (BTS) published guidelines supporting these findings, indicating 'stable' patients with PE could be managed in an outpatient setting. ${ }^{8}$

In order to identify patients with a low risk of an adverse outcome and therefore those potentially suitable for ambulatory care, Aujesky et al ${ }^{9,10}$ published a score to predict the severity of $\mathrm{PE}$, the Pulmonary Embolism Severity Index (PESI). The PESI is a points-based score, with points assigned for demographic characteristics, comorbid conditions and clinical severity markers., ${ }^{9,10}$ Individuals with a low total score that fell within 'Class I-2' severity were deemed low-risk as they had a $0.7 \%$ PE-specific mortality and I.2\% overall mortality three months after presentation. ${ }^{10}$ The authors proposed these low-risk patients were therefore potentially suitable for outpatient ambulatory care. 
In the setting of ambulatory care and therefore no immediate healthcare presence, potential adverse events, due either to the primary condition or to treatment are a cause for concern. Research by Ong et al investigated I 30 patients who underwent ambulatory treatment for PE. "Their selection criteria for ambulatory care included lack of haemodynamic instability, hypoxaemia (as defined by saturations $<90 \%$ ), intercurrent comorbidities or social factors impeding ambulatory care. In the study, $46 \%$ were treated exclusively as outpatients and $54 \%$ were treated as early discharge patients with an average hospital stay of 5.7 days. There were no fatalities during their first week of treatment. This is the period when patients would traditionally have been admitted for observation and initiation of oral anticoagulation. However, of 130 patients enrolled, two cases of major bleeding were identified in the first week. One of these patients bled from gastric ulcers and the second developed a pelvic haematoma. There were also 14 readmissions due to symptoms, bleeding and recurrent venous thromboembolism. There were no fatalities attributable to PE or its therapy in a three month followup period."

In 2006, Rowlinson et al published a year's experience with ambulatory investigation and treatment in Southampton, UK. ${ }^{2}$ They used slightly different criteria to exclude potentially high-risk patients unsuitable for outpatient care. These criteria included hypoxaemia (saturations $<97 \%$ ), clinical markers of haemodynamic instability, electrocardiogram evidence of right ventricular strain, symptoms at rest, or comorbid conditions (including underlying lung disease). ${ }^{2}$ Over a year, I 33 patients were identified as requiring investigation for $\mathrm{PE}$. Of these, 22 patients (16.5\%) were subsequently treated as outpatients for PE after imaging. One patient developed a vaginal vault haematoma after one dose of heparin but there were no other complications of anticoagulation. Eight patients were readmitted, but none of these had a confirmed PE and their readmission was felt to be due to persistence of their original symptoms. Five patients died during follow-up, but no death was found to be attributable to premature discharge or ambulatory care.

As previously mentioned, patient views, choices and satisfaction are central to any management plan and studies have shown that patients prefer ambulatory care. ${ }^{4}$ In 2006, a Rhodes et al study of 105 patients with PE treated as outpatients reported that $98 \%$ of patients would prefer outpatient ambulatory care if they were diagnosed with PE again. ${ }^{4}$ Davies (2007) corroborated this evidence with a survey showing that $96.6 \%$ of 157 patients treated for PE as outpatients would choose outpatient ambulatory treatment for any subsequent PE. ${ }^{2}$
At the Royal Infirmary of Edinburgh, the Emergency Department and Acute Medical Unit have been interested in increasing the role of ambulatory care, stimulated by the evidence and benefits described above. We developed a protocol designed to provide a pathway for managing patients presenting with possible PE (Figure I). Previously all patients would have been admitted pending investigation. Our pathway includes a probability score, determining the likelihood of PE using the major risk factors set out in the British Thoracic Society (BTS) guidelines. ${ }^{8}$ The second part, the change in our traditional management, was to establish suitability for ambulatory outpatient investigation if imaging was not available that day. This aimed to identify low-risk patients using a combination of the scores described above and specific factors contraindicating LMWH administration. We did not include ambulatory treatment for firmly diagnosed PE in this protocol - such a strategy is currently under development. The aim of this audit was to evaluate and report on the end-diagnoses, any adverse events and the resulting follow-up of patients managed on the ambulatory arm of this protocol for investigation of suspected PE.

\section{METHODS}

This open observational study and audit retrospectively investigated all patients who had been managed on the ambulatory arm of this protocol between December 2008 and April 2009. We identified patients by searching diaries in the Acute Medical Unit, in which staff booked in ambulatory patients returning for review (with or without imaging) to confirm or refute PE. Our interest was in the outcomes of patients in the ambulatory arm; as other patients not suitable for ambulatory management were admitted as a traditional safety default, they were not followed in this audit. The patients included in this audit met clinical probability for PE, positive D-dimer if required by the protocol (Instrumentation Laboratory D-dimer high sensitivity, cut-off $230 \mathrm{ng} / \mathrm{ml}$ ), and had no exclusion criteria for ambulatory management.

All end-diagnoses and any adverse events during the investigation period were determined by searching electronic patient records and discharge letters. The patient records covered all major secondary care hospitals in the Lothian region. Individualised follow-up contact was not attempted. The study looked into the records for subsequent management of ambulatory patients with confirmed PE by searching for admission or ambulatory anticoagulation with warfarin and then specialty follow-up arrangements.

The study was discussed with the local ethics department, who deemed the work an audit thereby not requiring National Health Service (NHS) ethical committee review. Observations were illustrated using summary statistics in the form of mean and median. 


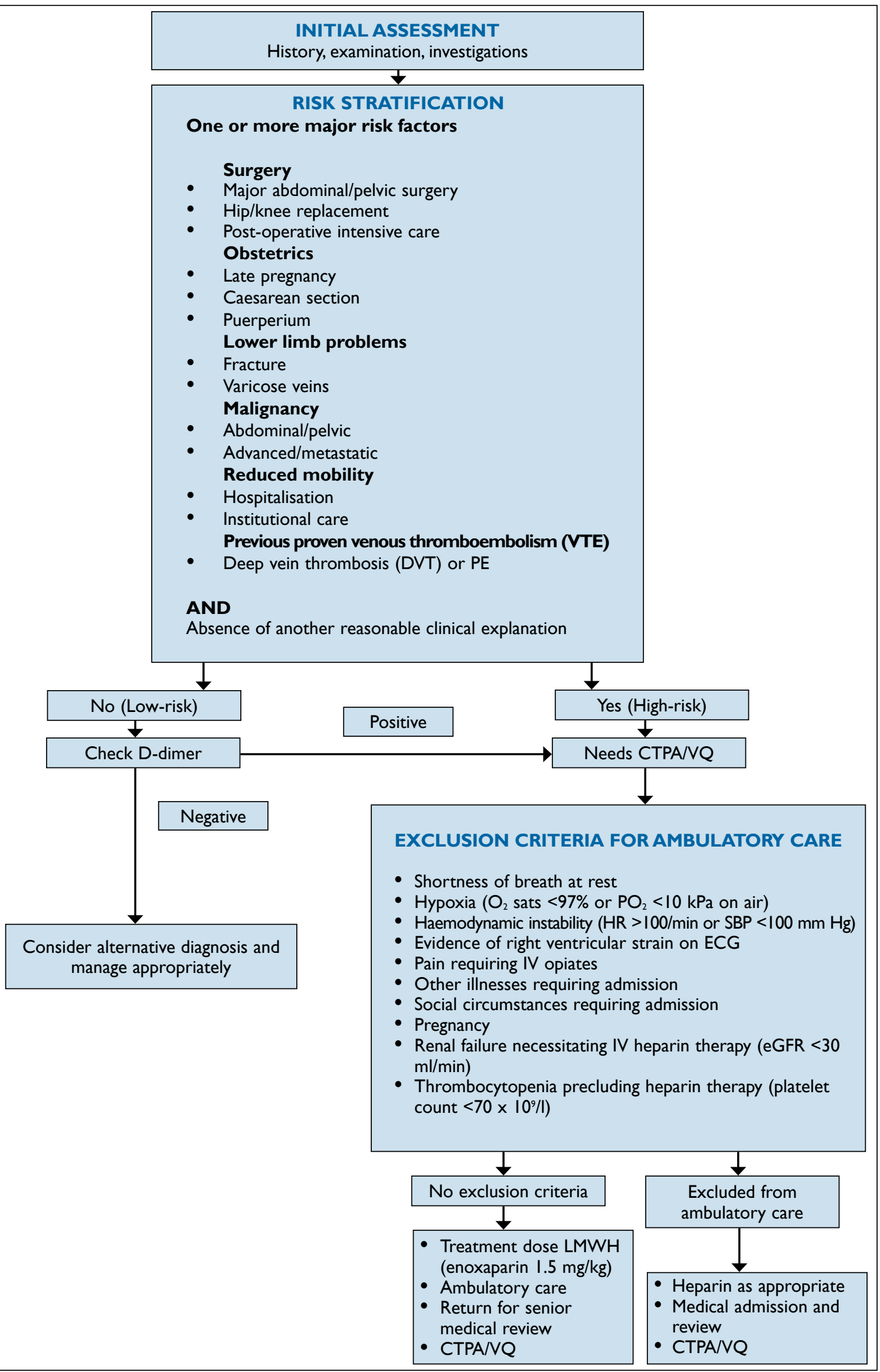

FIGURE I Protocol for ambulatory investigation of possible pulmonary embolism. 


\section{RESULTS}

In the five-month period of the audit, a total of 45 patients were managed in the ambulatory arm of this protocol. The mean age was 47.9 years (range 17-86) with a median of 44 . Thirty-one per cent of patients were male.

\section{Imaging}

After senior medical review, 41 (91.1\%) required radiological imaging. All patients received imaging within 24 hours. Thirteen patients were imaged by nuclear ventilation/perfusion (VQ) scan (28.8\%) and 28 required computed tomography pulmonary angiography (CTPA) (62.2\%) - one indeterminate VQ required CTPA confirmation. Five patients did not receive imaging due to very high clinical suspicion of $\mathrm{PE}$, the presence of an alternative diagnosis or discharge against medical advice despite fulfilling the criteria for investigation.

\section{Diagnoses}

Diagnoses were validated through clinical review of history and examination, in the context of presentation along with imaging findings. In $25 \%$ of patients, PE was confirmed. The next most common cause of symptoms was musculoskeletal chest pain (22.7\%). The next significant proportion of diagnoses was respiratory infection (15.9\%). As expected, CTPA was able to provide detailed structural imaging. Of the 28 scans, eight confirmed $\mathrm{PE}$, alternative diagnoses were provided in ten, one scan was indeterminate and no abnormalities, either suspected or incidental, were identified in the other nine cases. The case with an indeterminate scan was treated for PE on a clinical basis after further review and discussion with the patient.

\section{Adverse events}

During the period of ambulatory investigation, no patients managed on the ambulatory arm of this protocol had any adverse events or readmissions. While individualised contact was not attempted, no presentations to regional secondary care relating to $\mathrm{PE}$ or its management were noted for one month after ambulatory management.

\section{Follow-up}

Of the ambulatory patients diagnosed with PE, $72.7 \%$ were admitted for inpatient anticoagulation with warfarin. The remaining $27.3 \%$ were anticoagulated with low molecular weight heparin while initiating warfarin in ambulatory care as outpatients from the Medical Primary Assessment Area. All patients were followed up for review of their PE as outpatients, except one patient who was lost to follow-up. The specialties involved ranged between respiratory (seven patients), haematology, general medical outpatients and general practice (one patient each).

\section{DISCUSSION}

This audit has suggested that this management protocol can be applied to identify patients suitable for ambulatory investigation of PE. We were reassured that no adverse events were identified in relation to the protocol during our one-month record search. The audit showed that, using the data from the literature, a very low-risk group could be identified for ambulatory investigation. Even in this group with highly selective criteria, which might be expected to exclude the majority of PE, $25 \%$ of patients had PE confirmed. Given the significant proportion of patients who had PE confirmed, this study would support early treatment if imaging is not immediately available, as recommended in BTS guidelines. ${ }^{8}$ Our confirmation rate was moderately higher than previous studies such as Bauld et $\mathrm{al}^{13}$ and Rowlinson et $\mathrm{al}^{2}$, reporting $16.7 \%$ and $16.5 \%$ respectively, where fewer exclusion criteria were applied. However, neither of these studies used the BTS guidelines as a probability risk assessment, which may have a higher specificity for PE.

The protocol and its inclusion/exclusion criteria could be viewed as being overly stringent, especially as the PESI allocates risk points for oxygen saturations $<90 \%$, as opposed to the exclusion criterion of $<97 \%$ for our protocol. This in itself may have led to the relatively few numbers within the study group, a main study limitation. Rowlinson et al used an exclusion of $<97 \%$, ${ }^{2}$ and in the interests of identifying a very lowrisk group, this was the criterion included in our pathway. The protocol could be extended to encompass more patients by lowering oxygen saturation criterion to $94 \%$. This would allow inclusion of all patients who are not hypoxaemic, as defined in the 2008 BTS guidelines on emergency oxygen. ${ }^{14}$

A variety of alternative conditions can present in a manner similar to $\mathrm{PE}$, without obvious features to firmly guide the differential diagnosis. Formal identification of these on CTPA helps guide appropriate definitive management or further investigations as required (for example respiratory infection or pulmonary nodules). Our study indicated that in $8.9 \%$ of cases, senior clinical review of patients initially felt to have $\mathrm{PE}$ did not require imaging, as the review either revised the diagnosis to an alternative (three patients) or did not require imaging to confirm PE (one patient). For those who underwent imaging, CTPA was able to either confirm or exclude PE and provided alternative diagnoses in some cases. This has previously been recognised in work by Weiss et al in 2006 where $28.5 \%$ of 240 physicians surveyed in the US indicated CTPA had provided an alternative diagnosis to PE or showed significant abnormalities, which had then altered a patient's management. ${ }^{15}$ 


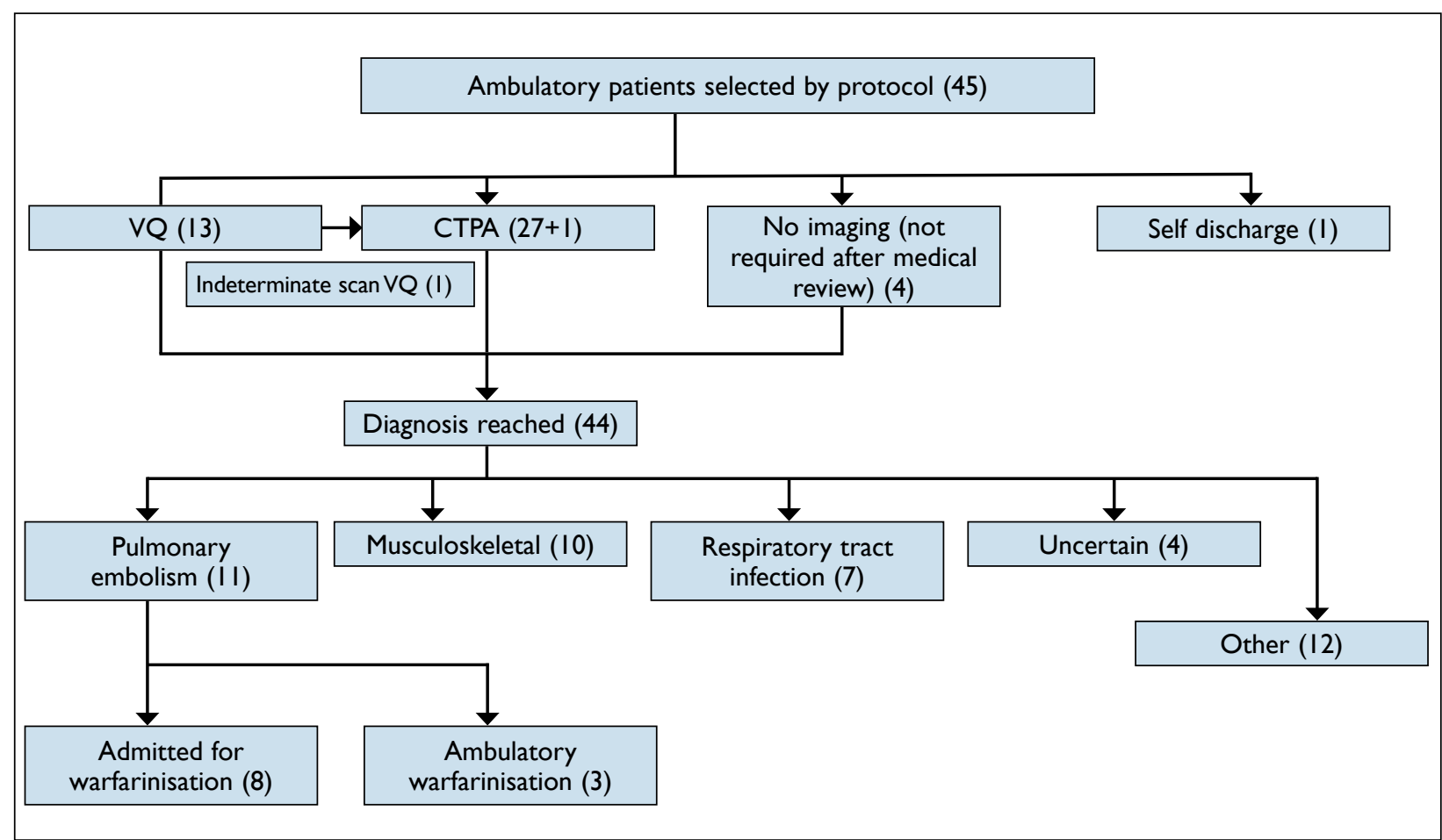

FIGURE 2 Flowchart indicating patient breakdown and numbers. 'Other' diagnoses include arrhythmia, biliary colic, costochondritis, pleural cystic change, symptomatic exertional breathlessness, oesophageal spasm, obstructive sleep apnoea, pleurisy, and pulmonary nodules.

This audit is limited by its open observational retrospective nature with small study numbers, and as such is subject to weakness and biases. Due to the design and nature of this audit, data are unavailable on the presentations and management of all patients presenting with possible PE including those who were then admitted pending confirmatory imaging (as would generally be our standard practice). We were only able to retrospectively study patients involved in the ambulatory care arm. A larger comparative prospective study of all patients presenting with possible PE would be required to accurately assess safety and effectiveness. In terms of follow-up, the audit was able to search electronic records, which cover the region's secondary care hospitals, but it did not collect data on any consultations in general practice, and we did not attempt individualised follow-up contact.

At the present time, no practical protocol exists in our hospital that defines selection for ambulatory anticoagulation with warfarin, nor is there a defined specific specialty follow-up arrangement. We therefore cannot draw conclusions regarding requirements for admission for anticoagulation or specific specialty follow-up.

This protocol was designed to select suitable low-risk patients for the investigation of possible pulmonary embolism where imaging was not available on the day. It does not investigate continuing ambulatory anticoagulation for patients diagnosed positively with PE.This audit therefore cannot comment on the safety of such a management strategy. We have also not studied the underlying cause for PE, but focused on its management. Acknowledging these limitations, the audit has been able to show the protocol's ability to select a low-risk population with possible $\mathrm{PE}$, and show a significant proportion of patients were diagnosed with PE despite the stringent exclusion criteria. We aim to restructure and extend the protocol to include practical selection criteria for ambulatory warfarin anticoagulation (as opposed to the generalised admission or early discharge for LMWH cover, pending therapeutic international normalised ratio).We hope this will provide a method of safely anticoagulating suitable patients without the need for admission. A larger comparative prospective study, potentially including the extension under development, is required to draw firmer conclusions on this pathway's safety and effectiveness.

\section{CONCLUSION}

There has been much interest in the ambulatory management of PE, and now literature exists to identify patients suitable for outpatient investigation. This audit shows a protocol can amalgamate this evidence and put it into practice. Our protocol was effective at selecting low-risk patients, and can be used for investigation and initial treatment of suspected PE where CTPA or VQ imaging is not available on the day. The protocol can reduce admission rates by providing an ambulatory 
method for investigation, which can cut costs while enhancing patient satisfaction. A larger comparative prospective study is now required to accurately assess the safety and effectiveness of this management protocol.

\section{ACKNOWLEDGEMENTS}

The authors would like to thank the JRCPE editorial team for their input and assistance.

\section{REFERENCES}

I Cohen AT,Agnelli G,Anderson FA et al.Venous Thromboembolism (VTE) in Europe. The number of VTE events and associated morbidity and mortality. Thromb Haemost 2007; 98:756-64.

2 Rowlinson JS, Deagle J, Roseveare CD. Ambulatory investigation and treatment of patients with suspected pulmonary embolism: a retrospective review of one year's experience. J $R$ Coll Physicians Edinb 2006; 36:12-16.

3 Goldhaber SZ, Visani L, De Rosa M. Acute pulmonary embolism: clinical outcomes in the International Cooperative Pulmonary Embolism Registry (ICOPER). Lancet 1999; 353:1386-89. doi:I0.10I6/S0I40-6736(98)07534-5

4 Rhodes S, Bond S. Shifting pulmonary embolism management to primary care. Nurs Times 2006; 102:23-24.

5 Griesbach D, Lardner C, Russell P. Managing the needs of drunk and incapable people in Scotland:A Literature review and needs assessment. Scottish Government Social Research; 2009. [cited 20II Apr 8] Available from: http://www.scotland.gov.uk/Publications/2009/ 10/29I54403/0

6 Kovacs MJ,Anderson D, Morrow B et al. Outpatient treatment of pulmonary embolism with dalteparin. Thromb Haemost 2000; 83:209-II.

7 Wells PS. Outpatient treatment of patients with deep-vein thrombosis or pulmonary embolism. Curr Opin Pulm Med 2001: 7:360-64. doi: 10.1097/00063198-200109000-00019

8 British Thoracic Society Standards of Care Committee Pulmonary Embolism Guideline Development Group. British Thoracic Society Guidelines for the management of suspected acute pulmonary embolism. Thorax 2003; 58:470-83. doi:10.1 I36/thorax.58.6.470
9 Aujesky D, Obrosky DS, Stone RA et al. Derivation and validation of a prognostic model for pulmonary embolism. Am J Respir Crit Care Med 2005; I72:I04I-46. doi:I0.I I64/rccm.200506-862OC

10 Aujesky D, Perrier A, Roy PM et al.Validation of a clinical prognostic model to identify low-risk patients with pulmonary embolism. J InternMed2007;26 I:597-604.doi:I0. I I I I/j.I365-2796.2007.0I785.x

II Ong BS, Karr MA, Chan DK et al. Management of pulmonary embolism in the home. Med J Aust 2005; 183:239-42.

12 Davies CW,Wimperis J, Green ES et al. Early discharge of patients with pulmonary embolism: a two-phase observational study. Eur Respir J 2007; 30:708-14. doi:10.1 I83/09031936.00140506

13 Bauld DL, Kovacs MJ. Dalteparin in emergency patients to prevent admission prior to investigation for venous thromboembolism. Am J Emerg Med 1999; I7: I I-I 5. doi:I0.1016/S0735-6757(99)90004-9

I4 O'Driscoll BR, Howard LS, Davison AG et al. British Thoracic Society guideline for emergency oxygen use in adult patients. Thorax 2008; 63:vil-68. doi:I0.II36/thx.2008.102947

15 Weiss CR, Scatarige JC, Diette GB et al. CT pulmonary angiography is the first-line imaging test for acute pulmonary embolism: a survey of US clinicians. Acad Radiol 2006; I3:434-46. doi:10.1016/j.acra.2006.01.002 